\title{
THE EFFECT OF AERODYNAMIC INTERFERENCE ON THE DYNAMIC RESPONSE OF PRISMATIC STRUCTURES
}

\author{
AHSAN KAREEM \\ Department of Civil Engineering, University of Houston, Houston, TX 77006 (U.S.A.)
}

(Received February 3, 1986; accepted in revised form October 7, 1986)

\section{Summary}

The interference and proximity effects on the dynamic response of prismatic bluff bodies are investigated in a series of wind tunnel tests. The results show that the presence of a single, or a pair of upstream prisms of the same size can increase the interaction factor. The effects are more pronounced in a boundary layer flow with a lower level of turbulence, especially near the reduced velocity of 5 . The measurements in the wake of the upstream prism suggest that in a boundary layer flow with low turbulence the wake has well-correlated fluctuations that cause adverse load effects on the downstream prism. The strength of these wake fluctuations is dampened by addition of incident turbulence. The study suggests that the mutual interference and proximity effects on the dynamics of tall buildings would be more pronounced for a group of tall buildings in coastal areas exposed to the oceanfront, followed by suburban locations and, to a lesser degree, urban areas.

\section{Introduction}

Predicting the dynamic behavior of prismatic bluff bodies in the presence of one, or a group of adjacent bluff bodies is a problem due to the lack of information available concerning the fundamental mechanics of the flow around bluff body groups immersed in turbulent boundary layer flows. Whilst the case of isolated bodies has received much attention, and there is growing understanding of their behavior in turbulent boundary layers, there remains a need for improving our qualitative as well as quantitative understanding of bluff body aerodynamics in the presence of adjacent bodies. In the case where several adjacent bluff bodies are exposed to a boundary layer flow, aerodynamic interference and interaction occurs in the flow-field. These interference effects result in either adverse or beneficial aerodynamic loading that can be identified as changes in the local pressure fluctuations or in the overall static as well as dynamic loading.

Within the last few years attention has increasingly been given to buffeting effects on neighboring tall buildings as a result of interference and proximity. These studies include some systematic investigations encompassing changes 
experienced in the local pressure fluctuations, static and dynamic force coefficients, aeroelastic response of prismatic building models and a variety of proprietary tests of particular topographical features and geometric layouts of surrounding structures [1-8]. These investigations have provided information for the development of a database to provide guidance in at least qualitatively estimating the level of interference for a select group of building geometries and their respective layout configurations.

The present study investigates the interference effects on the dynamics of bluff bodies of square cross-section in two approach boundary layers. The simulated boundary layers represent typical atmospheric boundary layers and the results of this study may provide guidance for the design of tall buildings exposed to open water and suburban flows. The range of reduced velocities in this study is much broader than most of those available in the literature. Furthermore, the wake fluctuations from the upstream buildings were monitored to obtain a quantitative explanation for the interference effects.

\section{Experimental}

Measurements were conducted in the meteorological wind tunnel at the Fluid Dynamics and Diffusion Laboratory, Colorado State University. A rigid, lightweight 3-in. square and 18-in. tall model, mounted on a flexible base to represent the fundamental mode in the two orthogonal directions of motion, was used in this study. All interacting bodies were made of polyurethane with an aluminum spline to duplicate the dynamic characteristics of the principal structure. All measurements were conducted for two approach flows, boundary layers 1 and 2, with the principal and interfering models mounted on a turntable with a large inertial mass at the downwind end of the long test section [2]. Boundary layers 1 and 2 are referred to as BL1 and BL2 for convenience. Flow over the floor of the test section was used to generate BL1, whereas for BL2, randomized roughness blocks were placed on the test section floor. A set of spires and a horizontal barrier at the entrance of the test section were also used to stimulate the growth of the boundary layers. The boundary layer BL2 represents suburban flow conditions. BL1 has a mean profile representing flow over open water, but the turbulence intensity is slightly lower than typical waterfront conditions at the upper third of the model height. These boundary layers were essentially used to delineate the influence of approach flow turbulence on the interference effects and associated dynamics of prismatic bluff bodies such as tall buildings. As such, BL1 data have the distinction of representing the only set of data available in the literature for this level of turbulence in a boundary layer flow.

A total of eighteen configurations were studied by monitoring the dynamic displacement at the top of the model with a single and a group of two obstructing models upstream of the principal model. The coordinates of these config- 
WIND

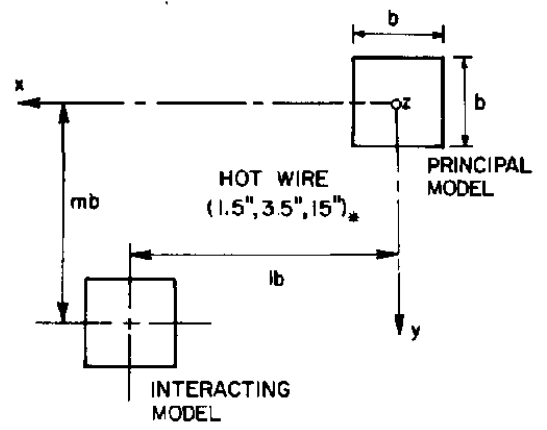

Fig. 1. Coordinate system and the location of hot-film.

urations are expressed in Fig. 1. The velocity fluctuations in the wake of the interacting prisms were monitored to identify the presence of any organized wake structure that may cause adverse load effects on the principal bodies (Fig. 1).

The outputs from the strain-gaged elastic base of the principal prisms and a constant-temperature hot-film anemometer were recorded using a digital data acquisition system. The important components of the instrumentation system are an analog-to-digital converter, a microcomputer and a digital tape-recorder. The statistical and spectral characteristics of the data were obtained based on time series analysis [2].

\section{Experimental results and discussion}

The mean velocity and local turbulence intensity profiles are presented as functions of non-dimensional height in Fig. 2 for the BL1 and BL2 flows, respectively.

The results of the interference and interaction due to adjacent models are presented in terms of interaction factors for a range of reduced velocities. The interaction factor (IF) is defined as

IF $=\frac{\text { r.m.s. displacement of the model with upstream model }}{\text { r.m.s. displacement of an isolated model }}$

Experimental results for only selected configurations consisting of a single and two upstream prisms are reported here. A detailed account of all tested configurations may be found in ref. 2 .

For a single upstream prism, offset laterally at two model widths, the interaction factors are reported in Fig. 3 . This configuration was relatively more severe than the upstream prism at the lateral offset of one model width. In Fig. 4 , results for a pair of upstream prismatic bluff bodies of the same dimensions for different lateral offsets and longitudinal separation distances are pre- 


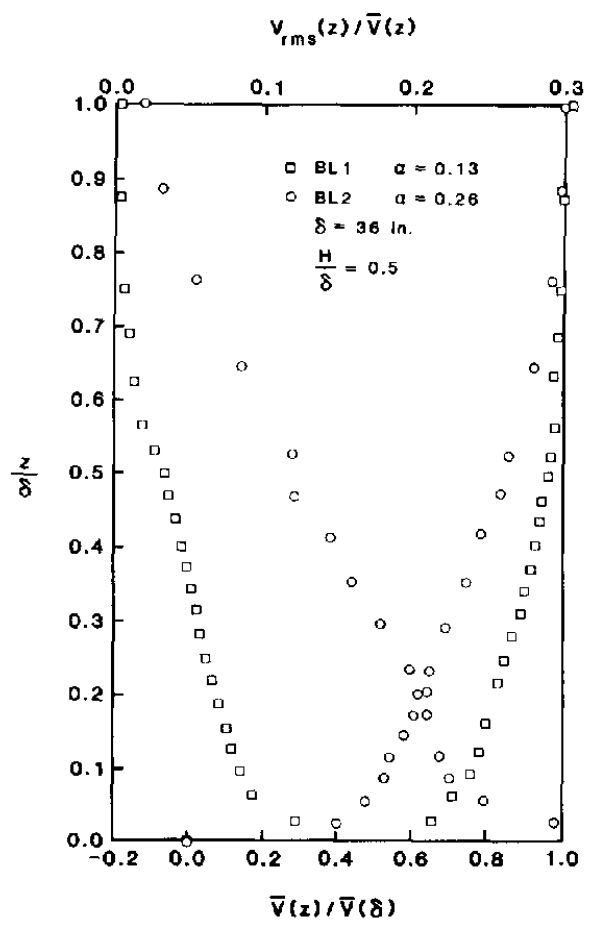

Fig. 2. Mean speed and turbulence intensity profiles for both boundary layers.

sented. These figures identify the coordinates of the upstream models. For both a single and a pair of upstream prisms the interaction factors are more significant in BL1 compared to BL2. Furthermore, IFs are more pronounced near the reduced velocity of 5 and gradually tend to decrease as the reduced velocity is increased. This trend is present for both the alongwind and acrosswind directions. One explanation for this trend may be attributed to the fact that at lower reduced velocities the response of an isolated prism in $\mathrm{BL} 1$ is much lower than the interference configuration. However, at higher reduced velocities the isolated prism's response increases, resulting in lower IF values. Similar trends in the IFs were observed by Sykes [7].

In Figs. 5 and 6 , the power spectra of the velocity fluctuations in the wake of the upstream prisms are shown for all the configurations presented in Figs. 3 and 4. The spectral description in Figs. 5 and 6 suggest that the level of turbulence intensity in the approach flow plays an important role in the interference mechanism. A low level of turbulence intensity promotes a wake behind an interacting model which has a high energy content in a narrow band of frequencies. The Strouhal number associated with the narrow band peak energy content is equal to 0.1 . These velocity fluctuations, in the wake of the upstream model, excite the principal model and lead to high values of IF. The addition 


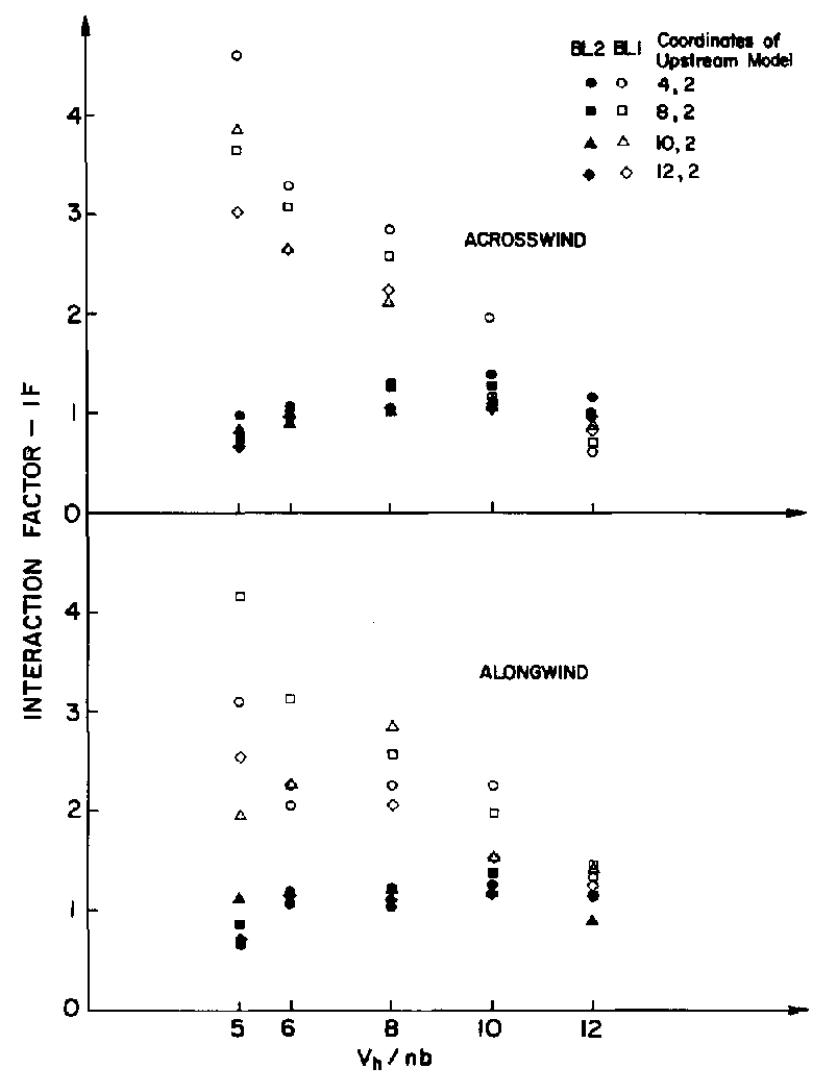

Fig. 3. Interaction factors for a single model upstream.

of turbulence redistributes energy in the wake velocity fluctuations of the upstream prism to a wider band of frequencies. This leads to lower levels of excitation for the principal prism resulting in lower values of IF in comparison with BL1 flow which has a lower turbulence intensity. In other words, if the fluctuating wind field in the wake is described by a wake-oscillator model, the addition of turbulence in the approach flow is analogous to the addition of damping in the wake-oscillator model, thereby dampening the wake fluctuations which are responsible for exciting the downstream prism.

\section{Conclusions}

The interference and proximity effects on the dynamic response of prismatic bluff bodies were investigated. The results of various configurations presented may provide guidance to the designers of prismatic tall buildings concerning the likelihood of adverse, or beneficial mutual interference and proximity effects 


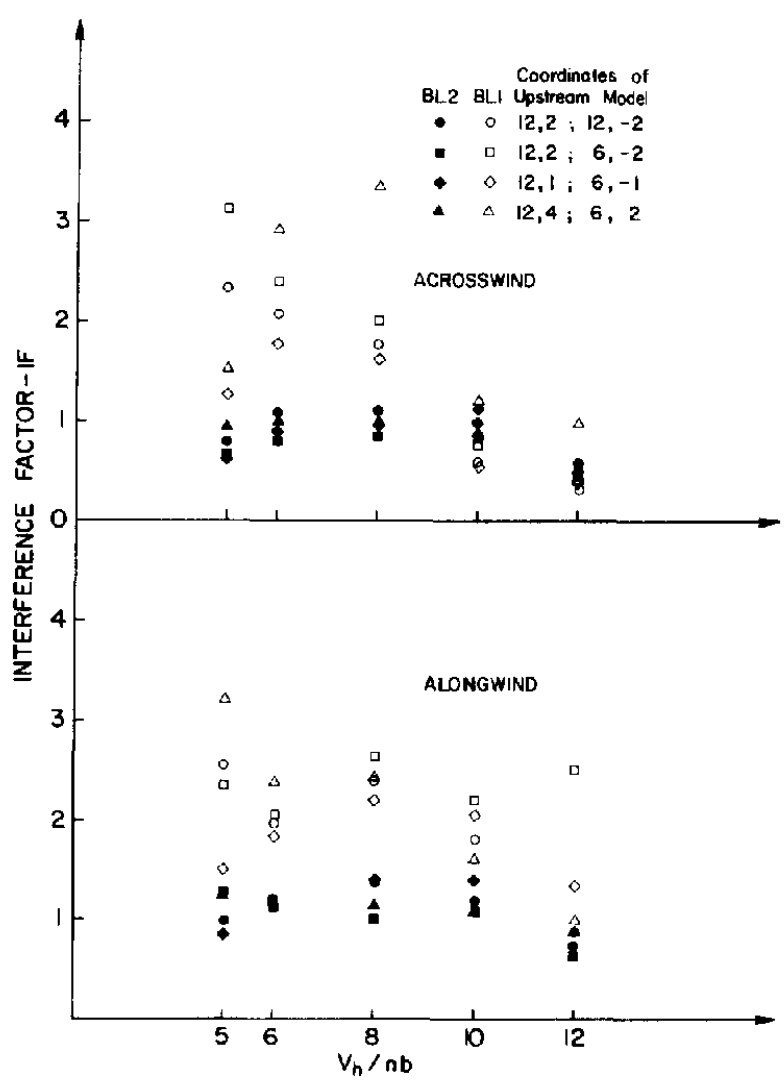

Fig. 4. Interaction factors for a pair of models upstream.

relating to buildings in suburban and waterfront locations. Some of the findings and the mechanisms of interference are summarized below:

(1) For both a single and a pair of upstream prisms, the interaction factors are more significant in the boundary layer flow with a lower level of turbulence. The IFs are more pronounced near the reduced velocity of 5 and gradually tend to reduce as the velocity is increased. These observations are valid for both the alongwind and acrosswind directions.

(2) The hot-film measurements in the wake of the upstream prism suggest that in a boundary layer flow with low turbulence the wake of the upstream prism has well-correlated fluctuations that cause adverse load effects on the downstream prisms. The strength of such wake fluctuations is dampened by the addition of incident turbulence, i.e., turbulence redistributes energy in the wake velocity fluctuations to a wider band of frequencies. Therefore, the mutual interference and proximity effects on the dynamics of tall buildings would be most pronounced for a group of tall buildings in coastal areas exposed to the oceanfront, followed by suburban locations and, to a lesser degree, urban areas. 


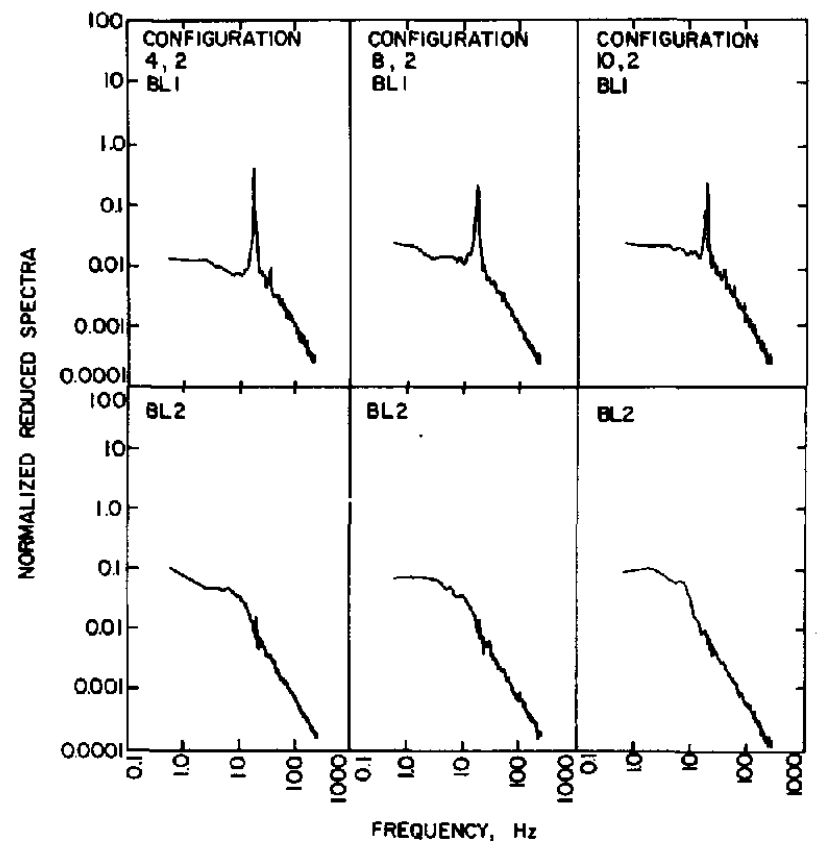

Fig. 5. Power spectra of wake fluctuations for a single upstream model.

Finally, the study was aimed at investigating the effect of aerodynamic interference and interactions on the overall dynamics of prismatic bluff bodies of equal height and plan dimensions. The results for different plan geometries

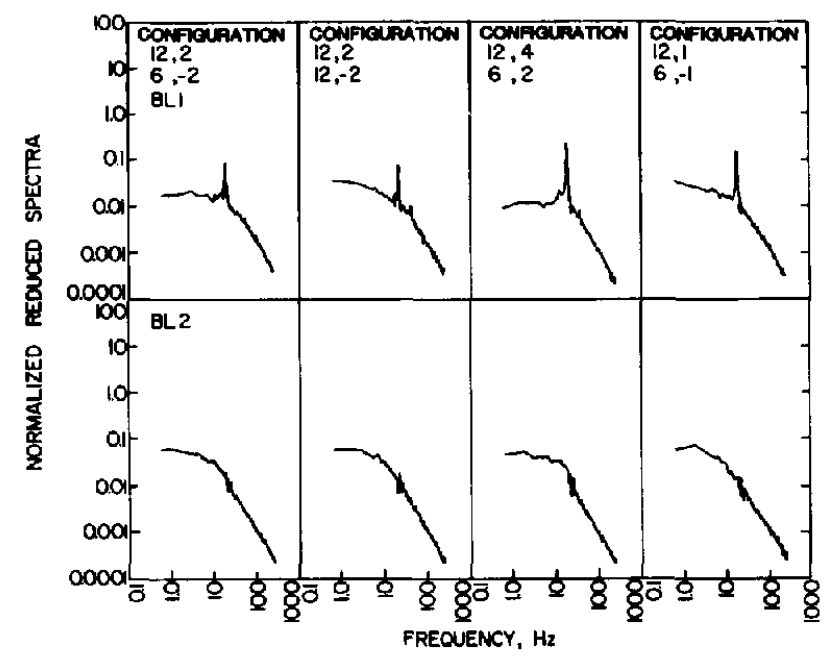

Fig. 6. Power spectra of wake fluctuations for a pair of upstream models. 
and heights may differ from those presented in this study. Furthermore, the magnitude of the localized fluctuating pressure on buildings, which was not investigated here, may be influenced differently under different boundary conditions due to interference and proximity effects.

\section{Acknowledgment}

The support for this study was provided in part by the National Science Foundation under Research Grants ENG-76-03135 and CEE-8019392.

\section{References}

1 J. Blessmann, Buffeting effects on neighboring tall buildings, J. Wind Eng. Ind. Aerodyn., 18 (1985) 105-110.

2 A. Kareem, Wind-excited response of building, Thesis, Colorado State University, Fort Collins, $\mathrm{CO}, 1978$, in partial fulfillment of the requirements for a $\mathrm{Ph} . \mathrm{D}$.

3 W.H. Melbourne and D.B. Sharp, Effects of upwind buildings on the response of tall buildings, Proc. Reg. Conf. on Tall Buildings, Hong Kong, Sept. 1976.

4 T.A. Reinhold and P.R. Sparks, The influence of an upstream structure on the dynamic response of a square-section tall building, Proc. 4th Colloq. on Ind. Aerodyn., Aachen, 1980.

$5 \mathrm{H}$. Ruscheweyh, Dynamic response of high rise buildings under wind action with interference effects from surrounding buildings of similar size, in J.E. Cermak (Ed.), Proc. 5th Int. Conf. on Wind Engineering, Fort Collins, CO, 1979, Vol. 2, Pergamon, Oxford, 1980, Pap. VI-6.

6 J.W. Saunders and W.H. Melbourne, Buffeting effects of upstream buildings, in J.E. Cermak (Ed.), Proc. 5th Int. Conf. on Wind Engineering, Fort Collins, CO, 1979, Vol. 1, Pergamon, Oxford, 1980, Pap. V-9.

7 D.M. Sykes, Interference effects on the response of a tall building model, J. Wind Eng. Ind. Aerodyn., 11 (1983) 365-380.

8 T.G. Zambrano et al., Wind-load interaction on an adjacent building, Proc. 3rd U.S. Natl. Conf. on Wind Engineering Research, University of Florida, Gainsville, FL, 1978. 